\title{
A Niched Pareto GA Approach for Scheduling Scientific Workflows in Wireless Grids
}

\author{
Shajulin Benedict ${ }^{1}$ and V. Vasudevan ${ }^{2}$ \\ ${ }^{1}$ Software Technologies Group, TIFAC Core in Network Engineering, AKCE \\ ${ }^{2}$ Director of Software Technologies Group, TIFAC Core in Network Engineering, AKCE
}

\begin{abstract}
We present a Niched Pareto Genetic Algorithm (NPGA) approach to the scheduling of scientific workflows in a wireless grid environment that connects computational resources, wired grids and wireless device resources such as cameras, microphones, network interfaces and so forth where the maximization of job completion ratio and minimization of lateness is crucial. Our approach supports handling uncertainty in the field of decision analysis, a rigorous technique for combining multiple objectives simultaneously. We made comparisons of our approach with respect to other scheduling policies; it performed significantly better than the majority of the cases, and in the worst cases, it was as good as the best of the others.
\end{abstract}

Keywords: genetic algorithm, grid computing, metaheuristic, niching, wireless grid.

\section{Introduction}

Wireless grids are now recognized as popular new economic sectors and even in a challenging time for the telecommunications industry, utilizing ubiquitous networks to access grid services. Fixed wireless grids borrow from the wired grid model using a variety of standards-based data transports methods combined in innovative ways. Mostly they pre-register an X.509 certificate before participation in Grid using Globus (http://www.wirelessgrids.net/). Its solutions offer home broad band users the maximum ability to share necessary documents, DVDs, music, movies, displays, cameras, printers, security devices, sensors and so forth. People increasingly take wireless devices [14] with them in both their personal and professional lives. It extends the vision of grid, framing an adaptive network with secure, inexpensive, co-ordinated real time access to dynamic heterogeneous resources that traverse geographical and political boundaries to sharing potential with mobile, nomadic or fixed location devices temporally connected via ad-hoc wireless networks.

However, most of the applications in wireless grids such as aggregating information of wireless devices, creating quality recording [9], locating nodes, data mining jobs, home automation and collection of nomadic devices, face the following challenges [7]:

- Unability to suit appropriate policies and radio technologies at the physical layer.

- Power consumption, efficient utilization of power, and coverage among the wireless networks.

- Most of the wireless devices are heterogeneous, low bandwidth and battery operated [8].

- Necessity for a prolonged period during handoff and disconnection.

- Multiple resource management and grid scheduling.

There is a variety of devices with diverse functionalities used for both personal and business utility. Hence sharing and aggregation of distributed resources increases the immediate need for mechanisms allowing an efficient management of limited resources. Following are the grid scheduling challenges: 
- Discovery mechanism and protocol design.

- Unpredictable challenges in grid resources (i.e., availability, accessibility, and so on).

- Security and policy management [10].

- Need for multiple resource types [13] for completing a job.

This paper addresses the problem of making sequencing and scheduling decisions for wireless grid scheduling problem environment. The availability of resources in grid sites is still limited, although the computers have reflected the growth in all extends, to run applications such as the mass spectrometric analysis in $3 \mathrm{D}$ rendering computations.

In considering the periodical jobs scheduled in advance, evolutionary techniques, namely, the Genetic Algorithm provides optimal results. In home automated wireless sensor grid sites tasks such as washing hands should be preceded by hand-drying task. This manifests that the tasks can be subdivided into workflow units and made as Directed Acyclic Graphs (DAG) or non Directed Acyclic Graphs (nDAG) as in parallel computations. The Genetic Algorithm is efficient in considering a single objective.

In this paper, it is attempted to employ Genetic Algorithm with Niching Pareto implementation in the Wireless Grid scheduler to solve the scheduling problem with multiple objectives for Wireless Grids. The multiple objectives such as maximum number of job completions and minimization of lateness for efficient scheduling are used. The evaluation of the Niching Pareto with other algorithms such as FCFS and EDF are made.

The rest of the paper is organized as follows: the next section discusses related works. Section 3 presents an overview of Wireless Intergrid scheduler Architecture and the environment where the NGPA is implemented. Section 4 describes the objective function and the Niched Pareto GA concepts. Results of the performance and parameter study are reported in Section 5.

\section{Related Works}

Recent years have seen many efforts focused on the efficient utilization of Grid resources by
Grid scheduling to appease the customer and provider satisfactions, such as Condor project [3], which being gradually developed for about fifteen years, aim for high throughput. The existing projects such as Alchemi aggregates the computing power of networked machines into a computational grid, Gridport aggregates service based applications, GridSim allows modeling and simulation of entities in the parallel and distributed computing systems, Gridway provides globus submission framework, Maui executes batch scheduling for high performance computing and so forth. Few projects namely Astrogrid, Avaki are orienting towards Data grids. Gridlab is a project aiming towards application development. Projects such as OptimalGrid and United Devices Metaprocessor Platform schedules and manages workloads considering load balancing. Albeit real time projects are emerging exponentially, the challenge towards Grid scheduling is succinct.

Two-level scheduling for large periodical jobs is discussed using the Genetic Algorithm in [15]. Here an optimal solution is selected using the parameters such as job characteristics, Grid environment characteristics and data distribution characteristics. In [11], scheduling two scientific workflows with the algorithm namely Heterogeneous Earliest Finish Time (HEFT) is compared with other myopic and genetic algorithms. HEFT schedules workflow by producing an order for the tasks. In [12], [13] scheduling scientific workflows using a multi-objective scheme and simulated annealing was worked out. Albeit, the algorithm obviously found optimistic results the discussion were for wired grids.

The works related to [1], consider intra- and inter-level grids for scheduling such that first level is used for mapping tasks among proxy nodes and the second level is used for scheduling among wireless domains. The work related to [4] has developed a new workflow model such that it maintains connection and state information to allow solid support for wireless applications. Each workflow has flow description, activity description and service description. This relates the DAG workflow model. The work done by [6] considers QoS management among the wireless devices by providing better information services. Our approach, using Niched Pareto Genetic Algorithm (NPGA) 
- based scheduling, is efficient for achieving a multiple objective nearer to optimal solution, especially in wireless grids.

\section{Problem Description}

Figure 1 shows the Wireless Inter-Grid Scheduler Architecture (WIGSA) with both wired and wireless grid users connected to it. This architecture could be used for any practical applications in the normal wireless grid environments.

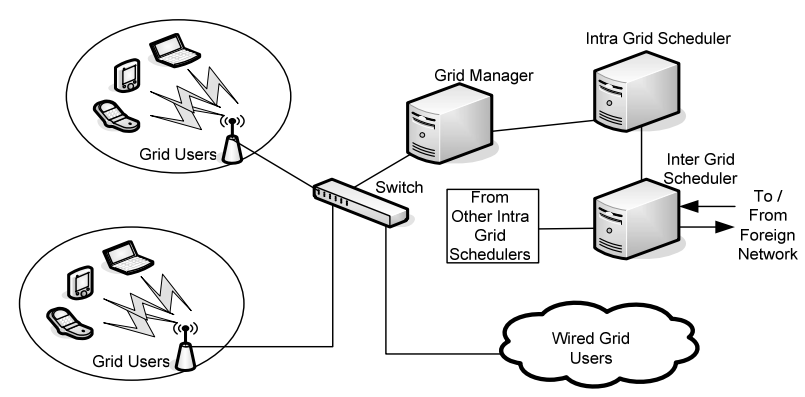

Figure 1. Wireles Inter-grid Scheduler Architechture.

The research is conducted in TIFAC Core in Network Engineering under the Department of Science and Technology funded project in India.

The goal of the WIGSA is to find the allocation sequence of workflows on each Grid site. Five major entities are involved in WIGSA:

- The Grid Users connected either via wired or wireless by Session Initiate Protocol [2] submit their requests for job completion as service requirements to the local grid managers.

- Each grid node has a sandbox to store the messages. The tasks should be received by the Grid managers and the decision for the scheduling is made on deploying the request to the Intra-grid schedulers.

- The Intra-grid schedulers authenticate, determines resource availability and checks for resource allocation within its local domain. It has the updated report of the wireless grid resources that are idle during time ' $t$ '. This information is frequently updated. The smaller jobs can be scheduled within the deadline by the Intra-grid schedulers.
- For missions critical and data intensive applications such as e-Health service, ECG application and Carotid artery application where the jobs are larger requiring the necessity of the limited resources that are computing intensive and placed globally. At that moment, there is necessary of Inter-Grid schedulers which are static often.

The goal of the Inter-grid Scheduler is to receive the request from different intra-grid schedulers and make an optimistic scheduling such that it accommodates many workflows completing within its deadline.

The simulator developed is to show the performance of the NPGA in comparison to the other traditional algorithms. The FCFS map tasks to the idle Grid sites based on first task arrival to serve first. The EDF algorithm executes the tasks whose absolute deadline are the earliest. Hence it estimates the execution deadline of the individual workflow for any standalone system and schedules such that the workflows that require greater completion time is served first. In EDF, the task priorities are not fixed but change depending on the closeness of their absolute deadline.

The setting of the experiment consists of workflows with the following assumptions:

- Each workflow received in the Inter-grid scheduler consists of a set of Tasks $T_{1}, T_{2}$, $T_{3}$, and so forth.

- The task in each workflow is a DAG or nDAG model.

- The output from a task can be transferred to other tasks as per the DAG graph model and its transmission time is negligible.

- At any time, a task can be executed only on a Grid site which is reported to the Inter-grid scheduler as idle via Intra-grid scheduler.

- There is no pre-emption of tasks or workflows.

The tasks taken for research have their predecessors and successors, such as $T_{1}$ follows $T_{2}$ or $T_{2}, T_{3}$ are parallel computation once the task $T_{1}$ is executed. For experimental purpose the following DAG workflows as given in Table 1 are considered.

The duration for each task in any workflow and the required grid resources are given in the Table 1 . 


\begin{tabular}{|l|l|l|c|}
\hline S.N & $\begin{array}{l}\text { Work } \\
\text { flow }\end{array}$ & \multicolumn{1}{|c|}{ Tasks/(duration) } & $\begin{array}{c}\text { Grid } \\
\text { Sites }\end{array}$ \\
\hline \hline 1 & $W 1$ & $\begin{array}{l}T_{1}(2), T_{2}(4), T_{3}(3), T_{4}(1), T_{5}(3), \\
T_{6}(2), T_{7}(3) .\end{array}$ & Any \\
\hline 2 & $W 2$ & $\begin{array}{l}T_{1}(3), T_{2}(2), T_{3}(3), T_{4}(4), T_{5}(3), \\
T_{6}(1) .\end{array}$ & Any \\
\hline 3 & W3 & $\begin{array}{l}T_{1}(2), T_{2}(4), T_{3}(1), T_{4}(2), T_{5}(3), \\
T_{6}(4), T_{7}(5) .\end{array}$ & Any \\
\hline 4 & $W 4$ & $\begin{array}{l}T_{1}(2), T_{2}(3), T_{3}(1), T_{4}(4), T_{5}(5), \\
T_{6}(1), T_{7}(2), T_{8}(3) .\end{array}$ & Any \\
\hline 5 & $W 5$ & $\begin{array}{l}T_{1}(1), T_{2}(3), T_{3}(2), T_{4}(4), T_{5}(5), \\
T_{6}(6) .\end{array}$ & Any \\
\hline 6 & $W 6$ & $\begin{array}{l}T_{1}(1), T_{2}(3), T_{3}(1), T_{4}(2), T_{5}(4), \\
T_{6}(2) .\end{array}$ & Any \\
\hline 7 & W7 & $\begin{array}{l}T_{1}(1), T_{2}(4), T_{3}(2), T_{4}(5), T_{5}(3), \\
T_{6}(2) .\end{array}$ & Any \\
\hline 8 & $W 8$ & $\begin{array}{l}T_{1}(1), T_{2}(3), T_{3}(2), T_{4}(3), T_{5}(1), \\
T_{6}(1), T_{7}(2), T_{8}(3) .\end{array}$ & Any \\
\hline 9 & $W 9$ & $\begin{array}{l}T_{1}(3), T_{2}(3), T_{3}(4), T_{4}(6), T_{5}(2), \\
T_{6}(4), T_{7}(2), T_{8}(1), T_{9}(5) .\end{array}$ & Any \\
\hline 10 & $W 10$ & $\begin{array}{l}T_{1}(4), T_{2}(5), T_{3}(4), T_{4}(4), T_{5}(5), \\
T_{6}(1), T_{7}(2) .\end{array}$ & Any \\
\hline
\end{tabular}

Table 1. Experimental Work Flows.

The workflow models for $W 1, W 5$ are shown as examples below:

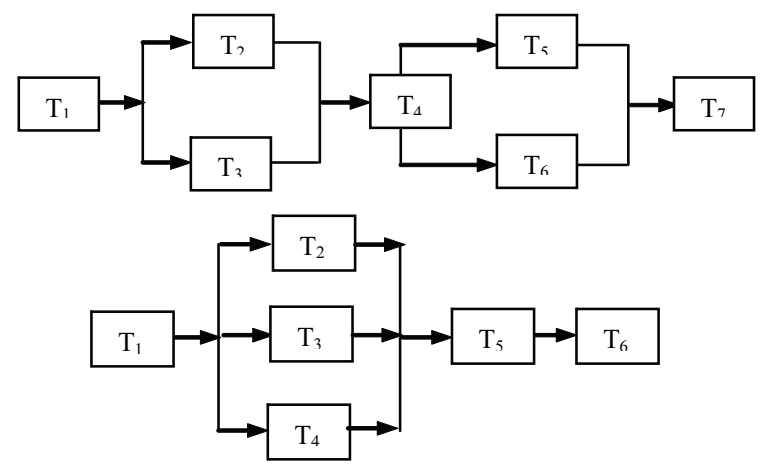

Figure 2. DAG for $W 1$ and $W 5$.

\section{The Niche based GA}

The Inter-grid scheduling for optimized scheduling, with the decision variable being the sequence of workflows to be fed into the Inter-grid scheduler and the constraint being the sequence of operations within a workflow with any available grid sites in the Grid environment, is as under:

\subsection{Simultaneous Objective Functions}

A standard Genetic algorithm works with a finite-length character string (chromosome) which represents the group of parameters of the significant problem. Niche-based GA has been developed to reduce the gradual effect of genetic drift resulting from the selection operator in the standard GA. They maintain the critical population diversity and permit the GA to investigate many objective functions in parallel.

The two objective functions used are as follows:

$F 1=$ Minimizing lateness of executing individual workflows:

$F 1=\sum_{i=1}^{m}\left(t w_{i}-d t w_{i}\right):$ only when $t w_{i} \geq d t w_{i}$

$F 2$ =Maximizing number of work flows completed with in the deadline:

$$
\begin{aligned}
F 2=\sum_{i=1}^{m} x w_{i} \quad \text { where } x w_{i} & =1 \forall t w_{i} \leq d t w_{i} \\
& =0 \text { otherwise. }
\end{aligned}
$$

\subsection{Coding Scheme}

The coding adapted for finding the practical solution is Pheno style as shown below:

$W 7, W 1, W 3, W 4, W 6, W 9, W 5, W 10, W 2, W 8$.

\subsection{Genetic Parameters}

The appropriate values of the GA parameters are arrived at based on the satisfactory performance of trials conducted for this application with different range of values. Population size $(\mathrm{n})=$ 20 samples; Crossover probability $(\mathrm{pc})=0.600$; Mutation probability $(\mathrm{pm})=0.005$; Termination criterion $=1000 \mathrm{GA}$ Cycles. The iterations make changes to the ranking and fitness values. 


\subsection{Reproduction}

Before the solutions are selected for the mating pool, solutions in the final population of the previous GA cycle are ranked assuming dominance with respect to the objective function. An Elite Group (EG) is constituted (with rank $\leq 3$ ) out of the Pareto ranked population.

The solutions obviously found in the Elite Group are niched using Fitness sharing method and their fitness values are degraded. Pareto dominance tournament selection method is employed on the current population with degraded fitness values, to create the mating pool.

\subsection{Pareto Ranking}

Every individual in the current population is compared with other individuals for dominance with respect to both objective functions $F 1$ and $F 2$. If an individual is non dominant over the rest of individuals with respect to both objective functions, it is given the first priority for the rank assignment. Otherwise, if it is non dominant over only one of the objective functions $(F 1$ (or) $F 2$ ) then it is given second priority for rank assignment. The procedure is repeated till all the individuals in the population are ranked. The Pseudo code for pareto ranking is explained in Figure 3 and based on the assigned ranks, the Elite Group is constituted with individuals having pareto ranking $\leq 3$.

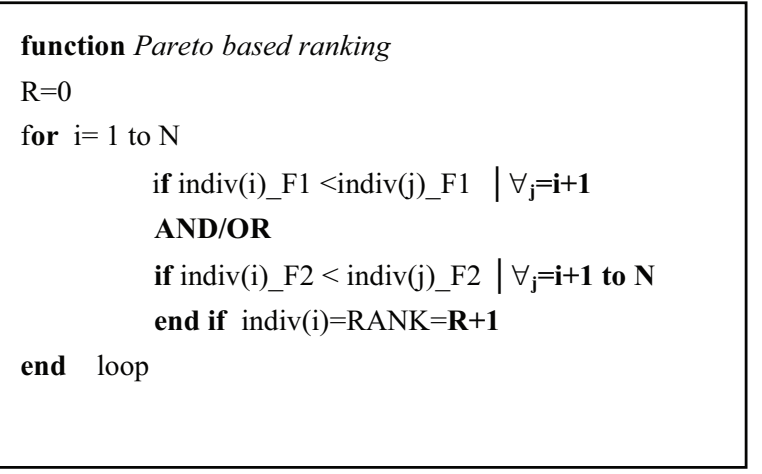

Figure 3. Pseudo code for pareto-based ranking.

\subsection{Pareto Tournament Selection}

The individuals for the mating pool are selected based on Pareto tournament selection schemes. Two candidates are selected at random from the population as comparison set. Repeatedly, another two individuals are drawn from the remaining population as reference set. If one candidate from the reference set to dominate both individuals of comparison set while other is not, latter is selected and carefully placed in the mating pool. If the mating pool is not filled with enough individuals, another random comparison set is drawn from the population and pareto tournament is repeated. By this method, the best individuals are allowed to have a high probability of being represented in the next population.

\subsection{Fitness Sharing}

Fitness sharing method [5] of niching is assigned on the individuals in the Elite Group to degrade their fitness value, proportionate to the number of individuals closer to it. The domination of individuals can cause the niching GA to converge prematurely.

To prevent premature convergence, simulated annealing during the pursuit search can be used. A triangular sharing function with values ranging from 1 to 0 is employed.

This modifies the search landscape by reducing the payoff in densely populated regions. It lowers each population element's fitness by an amount nearly equal to the original number of similar individuals in the population.

The pseudo code for niching as shown in Figure 4 below:

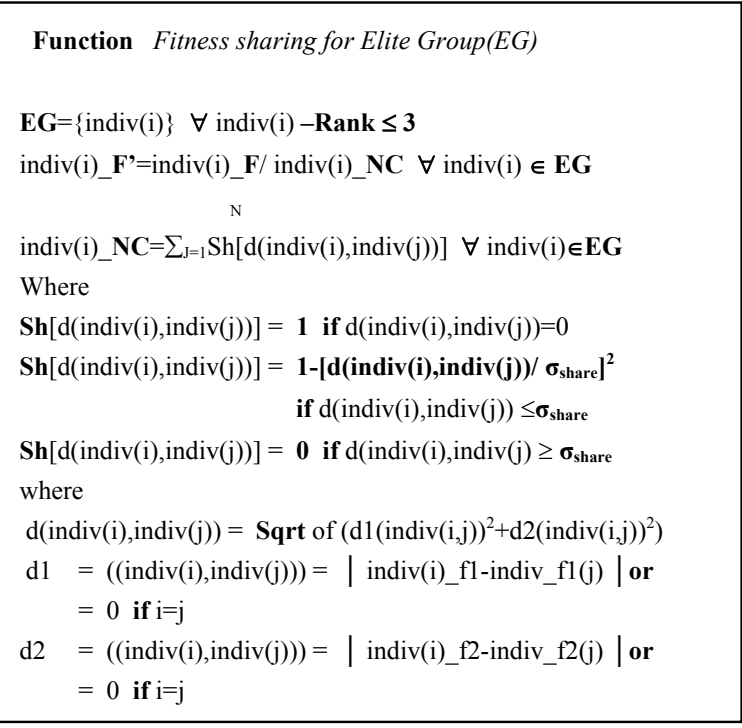

Figure 4. Pseudo code for fitness sharing. 


\subsection{Crossover}

A pair of strings is selected with the probability of cross over pc and crossed at a random site. For example, if the random site is 5th position, then strings are crossed after the 5th position. After the 5th position, the sequence of parts in the first string of the pair is rearranged according to the order of their outward appearance found in the second string. Similarly, after the 5th position, the sequence of parts in the second string of the pair is rearranged according to the order of their appearance found in the first string. The tuning of the crossover probability has been obtained with several tests run ranging from 7 to 30 workflows.

Example: $($ Crossover site $=5$ th position $)$

\section{Pair of strings before crossover}

$W 6, W 2, W 7, W 5, \underline{W 1}, W 4, W 3, W 8, W 10, W 9$. $W 7, W 1, W 4, W 10, \underline{W 9}, W 2, W 5, W 6, W 3, W 8$.

\section{Pair of strings after crossover}

$W 6, W 2, W 7, W 5, \underline{W 1}, W 4, W 10, W 9, W 3, W 8$. $W 7, W 1, W 4, W 10, \underline{W 9}, W 6, W 2, W 5, W 3, W 8$.

\subsection{Mutation}

Two sites are selected by generating two random numbers between 1 and 10. For example, if the generated random numbers are 2 and 8 , then the corresponding part numbers in these positions are interchanged and the new sequence is obtained,

\section{String before mutation}

$W 6, \underline{W 2}, W 7, W 5, W 1, W 4, W 10, \underline{W 9}, W 3, W 8$

\section{String after mutation}

$W 6, \underline{W 9}, W 7, W 5, W 1, W 4, W 10, \underline{W 2}, W 3, W 8$

\section{Results and Discussion}

This optimal schedule for the Wireless Intergrid computation of workflows is obtained by the technique using NPGA. This is compared with sequences obtained by different scheduling rules viz. FCFS, and EDF. For the experimental problem, the sequence obtained by NPGA gives minimum lateness for each workflow received in Inter-grid schedulers and maximize the job completion ratio (JCR). Hence it outperforms all the previous procedures.

The maximum number of workflows completed by different algorithms such as FCFS, EDF, and the NPGA is disclosed in Figure 5.

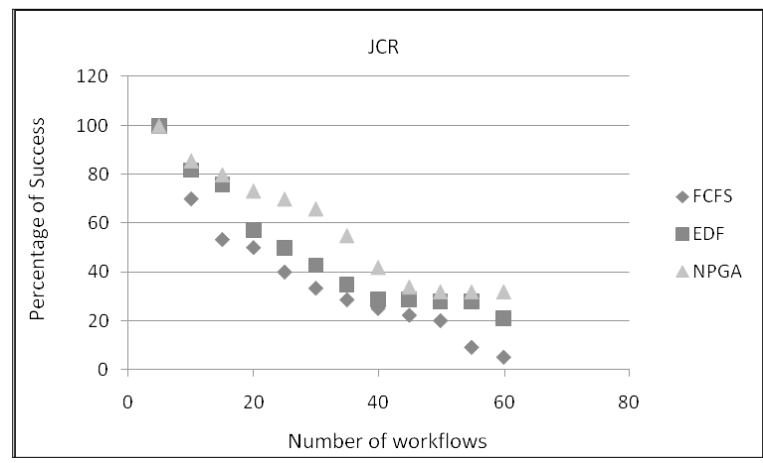

Figure 5. Job completion ratio (JCR).

The lateness for the completion of workflows minimizes with NPGA scheduling algorithms. Figure 6 reveals the minimization of lateness of the scheduling scheme.

As shown, the lateness increases abruptly for the scheduling schemes with FCFS and EDF algorithms. NPGA provide a near optimal solution much faster, which remains more important for the data mission critical wireless applications. The result manifests the crucially of the NPGA algorithm since it is obvious that the lateness by other algorithms is unaffordable. On suitably choosing the Pareto ranking, the algorithm outperforms for its better execution time within deadlines.

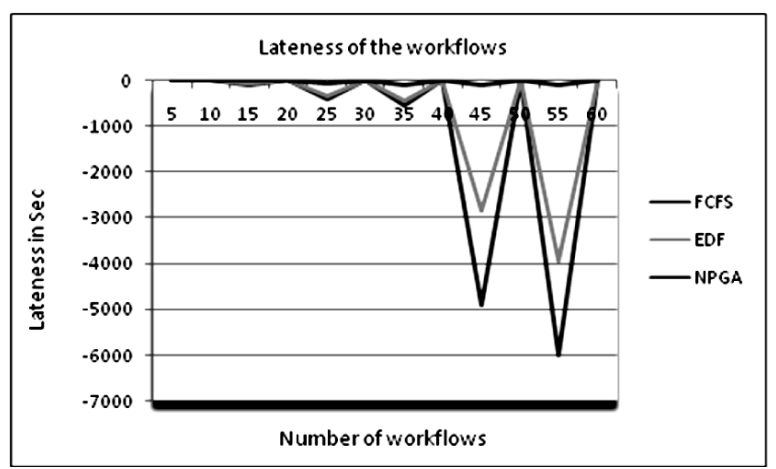

Figure 6. Lateness of the workflows. 


\section{Conclusion}

The Niched Pareto Genetic Algorithm has been described from the perspective of scheduling scientific workflows with simultaneous optimization of the dual objectives of a Wireless Inter-grid Scheduler. Possible wireless grids such as homogenous grid, intra-grid and intergrid can be deployed to provide data, computing and utility services. The NPGA procedure is tested with an example problem environment and the results are compared with that of other scheduling procedures. It is found that NPGA is more effective than the other algorithms.

In the near future, we shall combine simulated annealing along with the sharing method to increase the effectiveness. Similarly, the ant colony properties can be included for scalable in the existing algorithm. The procedure could also be suitably modified and applied to explore new mechanisms to build more secure, robust and energy efficient scheduling of workflows.

\section{Acknowledgment}

This work was supported by the Department of Science and Technology funded project in TIFAC Core of Network Engineering. We would like to thank our Project Coordinator Software Technologies Group for helping us start and continue this exhausting work.

\section{References}

[1] A. AgARWAL ET AL, Wireless grids: Approaches, architectures, and technical challenges. 2004, pp. $1-9$.

http: //ssm. com/abstract $=489782$

[2] FANG - YIE LEU ET AL, A Wireless Grid Service Platform Using SIP and Agents. In Proc. of the Seventh ACIS Int. Conf. on Software Engg, (SNPD'06), (2006), pp. 139-144.

[3] J. Frey, T. TAnnenbaum et AL, Condor-G: A Computation Management Agent for Multi-Instituitional Grids. Journal of Cluster Computing, 5, (2002), pp. 237-246.
[4] H. GuO, M. YANG ET AL, Autonomous and Dynamic Web Service Composition in Wireless Grids. In Proc. of Int. Conf. on Information Tech. Coding and Computing, (2005), pp. 574-579.

[5] J. Horn, N. Nafploitis, D. E. GoldberG, A Niched Pareto Genetic Algorithm for Multi Objective Optimization. Proceedings of the First IEEE International Conference on Evolutionary Computation, IEEE Press, Piscataway, (1994), pp. 82-87, NJ.

[6] J. YANG ET AL, WGMDS: A WSRF - Compliant Information Service on Wireless Grids. In Proc. of Int. Conf. on PDCAT' 05, (2005), pp. 103-105.

[7] J. Hwang, P. ARAVAmUdHAM, Middleware Services for P2P Computing in Wireless Grid Networks. Internet computing, (August 2004), pp. 40-47.

[8] L. W. MCKNIGHT, J. Howison, Towards a sharing protocol for wireless grids. In the Proc. of CCCT'03, 000648, (2003).

[9] L. W. MCKNiGHT, J. Howison, S. BRADNER, Wireless Grids: Distributed Resource Sharing by Mobile, Nomadic and Fixed Devices. IEEE Internet computing, (August 2004), pp. 24-32.

[10] L. W. McKnight, D. AniUs, O. Uzuner, Virtual Markets in Wireless Grids: Peering Policy Obsacles.

http://ebusiness.mit.edu.

[11] M. WIECZOEK, R. ProdAn, T. FAHrInGER, Scheduling of Scientific Workflows in the ASKALON Grid Enivironment. SIGMOD Record, 34(3), pp. 56-62, September 2005.

[12] S. BenEDICT ET AL, Scheduling Scientific Workflows Using Niched Pareto GA for Grids. In Proc of IEEE Int. Conf. on Services, Operations, Logistics, INSPEC, (June 2006), pp. 902-912.

[13] S. BenEDICT ET AL, Scheduling scientific workflows using simulated annealing for computational grids. In International Journal of Soft computing 2(5), (2007), pp. 606-611.

[14] S. H. SRINIVASAN, Pervasive wireless grid applications. In Proc. of 2nd Annual Conf. on Wireless on Demand Network Systems and Services (WONS'05), (2005), pp. 83-88.

[15] V. Di Martino, M. Mililotti, Scheduling in a Grid computing environment using Genetic Algorithms. Proceedings of the Parallel and Distributed Processing Symposium, (2002), pp. 235-239. 
Received: September, 2007

Accepted: November,2007

Contact addresses:

Shajulin Benedict

Software Technologies Lab

Tifac Core in Network Engineering

Sirivilliputhur

India-626190

e-mail: shajulinbenedict@akce.ac.in

Dr. V. Vasudevan

Director, Software Technologies Lab

TIFAC Core in Network Engineering

Sirivilliputhur

India-626190

SHAJULIN BENEDICT graduated in 2001 from the Electronics and Communication Engineering Department at the Manonmaniam Sunderanar University, India, with Distinction. In 2004, he received M.E Degree in Digital Communication and Computer Networking from Anna University, Chennai. He is the University second rank holder for his masters. He is working towards the Ph.D degree in the area of Grid scheduling. He is affiliated towards the Software Technologies Group of TIFAC Core in Network Engineering as Project Engineer, Sirivilliputhur, India. His research interests include Grid scheduling, Service Level Agreement, Agent Technology, Optimal routing in Grids, and so forth.

DR. V. VASUDEVAN received his $\mathrm{PhD}$ degree from the Manonmaniam Sunderanar University. He did his B. Sc in Mathematics and worked for several areas towards Representation Theory since 1986 . He is now the Project Director of the Software Technologies Group of TIFAC Core in Network Engineering and Head of the Department for Information Technology at Kalasalingam University, Sirivilliputhur, India. His research interests include Grid computing, Agent Technology, Intrusion Detection system, Multicasting and so forth. 